\title{
Postmortem quantification of brain edema by determination of electromagnetic tissue parameters
}

\author{
T. Reinecke $^{1}$, L. Hagemeier ${ }^{2}$, V. Schulte ${ }^{1}$, H. Spehlbrink ${ }^{1}$, M. Klintschar ${ }^{2}$, S. Zimmermann ${ }^{1}$ \\ ${ }_{1}^{1}$ Institute of Electrical Engineering and Measurement Technology, Department of Sensors and \\ Measurement Technology, Leibniz University, Hannover, Germany \\ reinecke@geml.uni-hannover.de \\ ${ }^{2}$ Institute of Forensic Medicine, Hannover Medical School, Hannover, Germany
}

\begin{abstract}
One important task during autopsy is the evaluation of edema, since the occurrence of edema, especially in the brain, is often directly linked to the cause of death. However, there is still no standardized procedure in forensic medicine to exactly quantify edema in parenchymatous organs. Thus the aim of our work is to explore the possibilities to determine the dielectric constant of brain tissue that correlates with the tissue's humidity content. Based on these results, the development of a fast and accurate, but low-cost measurement system that fits into the procedure of an autopsy is aspired. Our first approach is based on a coaxial measurement of the complex permittivity in order to investigate the relation between permittivity and humidity content. The humidity content was measured with a thermo gravimetric reference system. We found a linear regression with an excellent correlation coefficient between the real part of the permittivity and the humidity content in the tissue. These results show that an objective quantification of edema via a measurement of the complex permittivity is possible with a high accuracy. The subsequent step is the usage of a so-called open-ended coaxial line for the determination of the tissue's permittivity to avoid a sampling process und thus to speed up the measurement procedure. Here, we present first measurement results that are in good agreement with the results gained from the previous used coaxial measurement setup.
\end{abstract}

Key words: Permittivity of brain tissue, dielectric constant, aquametrie, open-ended coaxial probe.

\section{Introduction}

The humidity content in the brain is strongly dependent on the cause of death [1]. In case of a slow death, for example a lethal intoxication, the brain expands and thus creates edema. Contrary, an immediate death, such as a railway suicide, will cause practically no edema, because all bodily functions are stopped fast leaving no time for the brain to expand, even though a trauma of the brain occurred. Therefore, the exact measurement of the humidity content in certain brain regions might be an excellent marker to distinguish between causes of death where no apparent reason is visional, for example sudden infant death syndrome versus shaken impact syndrome. In current autopsies, the evaluation of edema in the human brain is done indirectly by examining brain-weight, turgor or ventricle compression, which is prone to errors.

One method for the determination of the humidity content is the so-called oven dry method where a specimen is heated up by microwaves, until all containing water is evaporated. Then, its humidity content is calculated from the weight difference before and after evaporation [2]. This procedure is very exact, but has the major drawback of expensive and bulky equipment. Additionally, the measuring process can take several minutes for one sample due to sample preparation and the time needed for the evaporation process, so that a systematic scanning for edema is timeconsuming. Therefore, the aim of our work is the development of a small, low-cost, but fast and accurate measuring system that fits into the procedure of an autopsy. Therefor we utilize the quantitative dependency of the permittivity of a material and its humidity content [3]. The first approach is based on a coaxial measuring system, which is calibrated with the oven dry method, so that a quantitative determination of the humidity content via a permittivity measurement is possible. Based on these results a so-called open-ended coaxial method is tested for humidity content determination in tissue. 


\section{Coaxial measurement system}

The theory for determining the permittivity of a material in a coaxial line is described in [4]. The associated schematic measurement setup is depicted in Fig. 1. The specimen is inserted as a dielectric into a coaxial line. The impedance of this coaxial line depends on the dielectric, so that the impedance of the specimen-filled part of the coaxial line generally differs from the impedance of the unloaded coaxial line. The waves propagating in such a coaxial line are so-called transverse electromagnetic (TEM) waves.

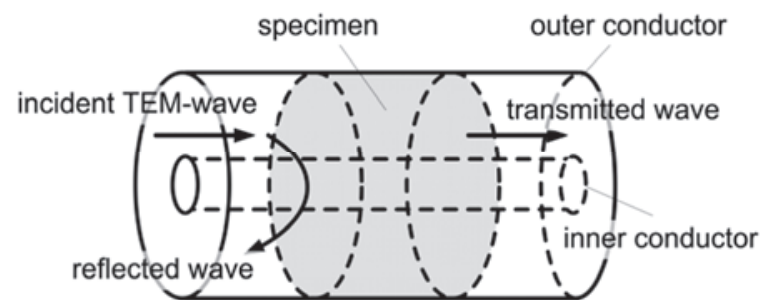

Fig. 1: Schematic of a coaxial line filled with a specimen as dielectric between the inner and outer conductor.

If such a wave interacts with a change of impedance at the interface of the specimenfilled part of the line, it is partly reflected and partly transmitted through the specimen. Thus, the specimen can be interpreted as a transmission line of length $l$ with the transmission coefficient

$$
z=e^{j \cdot \frac{\omega}{c_{0}} \cdot \sqrt{\varepsilon_{r} \mu_{r}} \cdot l}
$$

and the reflection coefficient

$$
\Gamma=\frac{\sqrt{\frac{\mu_{r}}{\varepsilon_{r}}}-1}{\sqrt{\frac{\mu_{r}}{\varepsilon_{r}}}+1} .
$$

Here $\omega=2 \pi f$ is the angular frequency, $\mathrm{c}_{0}$ the speed of light, $\varepsilon_{r}=\varepsilon_{r}^{\prime}-j \varepsilon_{r}^{\prime \prime}$ the complex relative permittivity and $\mu_{r}=\mu_{r}^{\prime}-j \mu_{r}^{\prime \prime}$ the complex relative permeability of the material.

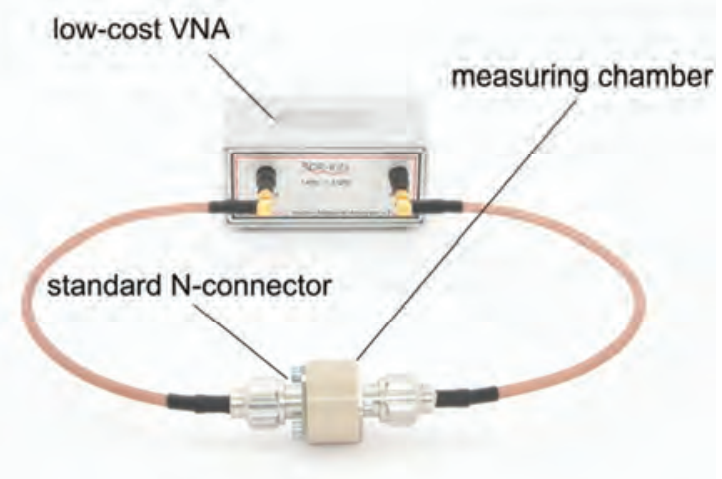

Fig. 2: Measuring setup with VNA (VNWA3, SDRKits) and measuring chamber
With $\Gamma$ and $z$, the interaction of a TEM-wave with the specimen can be described mathematically with the so-called scattering parameters. The scattering parameters can be seen as transfer functions from an incident wave $a_{1}$ to a reflected Wave $b_{1}$ as

$$
S_{11}=\frac{b_{1}}{a_{1}}=\frac{\left(1-z^{2}\right) \Gamma}{1-\Gamma^{2} z^{2}}
$$

and from $a_{1}$ to a transmitted Wave $b_{2}$ as

$$
S_{21}=\frac{b_{2}}{a_{1}}=\frac{\left(1-\Gamma^{2}\right) z}{1-\Gamma^{2} z^{2}}
$$

respectively. The equations (3) and (4) can be solved directly for the complex material parameters $\varepsilon_{r}$ and $\mu_{r}$.

But as described in [5] this direct solution shows an unstable behavior at multiple integers of the half wavelength in the sample. For nonmagnetic materials $\left(\mu_{r}=1\right)$, such as brain tissue, an alternative, stable solution can be found by expressing (3) as

$$
0=\frac{\left(1-z^{2}\right) \Gamma}{1-\Gamma^{2} z^{2}}-S_{21}
$$

The relative permittivity is found by applying a root finding algorithm to (5).

Fig. 2 shows the complete measuring setup consisting of a commercially available low-cost vector-network analyzer (VNA) VNWA3 (SDRKits) and our measuring chamber. The measuring chamber is easy to assemble and dissemble and consists of a housing, an inner and outer conductor and two modified $\mathrm{N}$ connectors. The outer conductor can be used as a die cutter for an easy and fast tissuesampling process as depicted in Fig. 3.

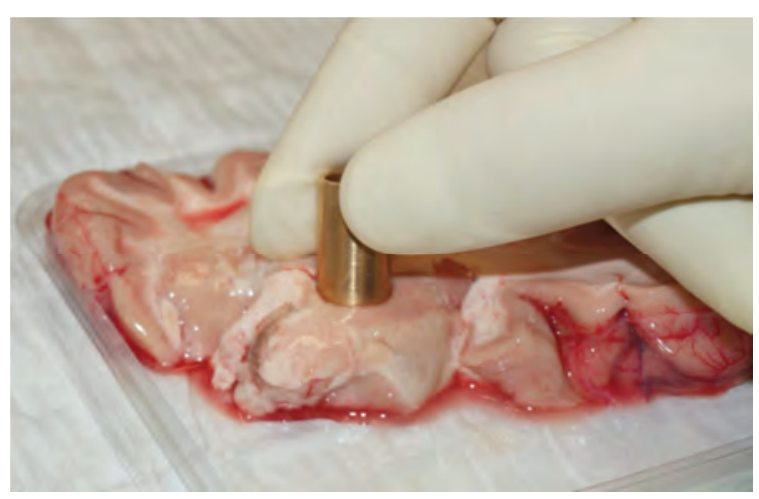

Fig. 3: Sampling the Thalamus

After sampling the outer conductor is inserted back into the housing so that the inner conductor punctures the specimen centrically, avoiding air inclusion or damaging of the specimen as depicted in Fig. 4. 


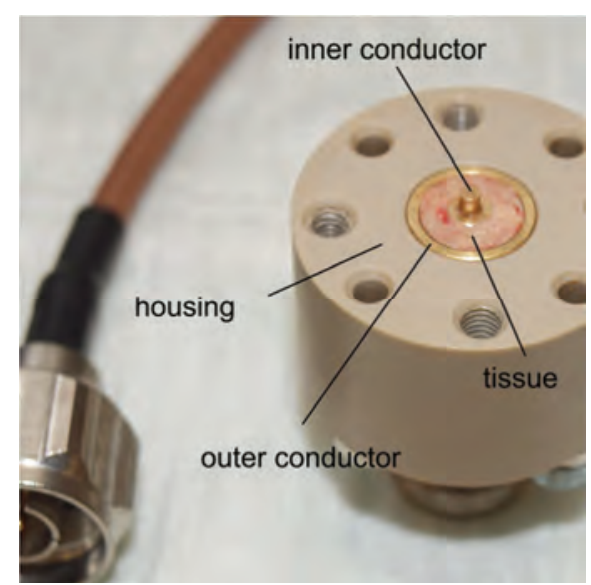

Fig. 4: Filled measuring chamber

As shown in [6], we performed measurements of the real part of the permittivity of brain tissue samples from six different probates and different brain regions with the developed system in the frequency range from $400 \mathrm{MHz}$ to $500 \mathrm{MHz}$. The humidity content of these samples was determined with the described oven dry method using the commercially available thermo gravimetric system Smart System5 ${ }^{\mathrm{TM}} \quad(\mathrm{CEM} 囚)$. Therefore, the tissue sample is applied on a glass fiber sample pad and put on a balance pan in the microwave chamber of the moisture analyzer. The specimen is then heated by microwaves with a power of $150 \mathrm{~W}-300 \mathrm{~W}$. The heating process is actively controlled, so that the water is evaporated without burning the tissue sample. The sample weight is monitored online during the heating process. When the weight is constant, because all water is evaporated, the heating program stops and the humidity content is determined by the weight difference before and after evaporation.

Fig. 5 shows the real part of the permittivity of the measured brain tissue samples at a frequency of $490 \mathrm{MHz}$ as a function of the humidity content of the samples. The plot shows a linear regression between the permittivity and the humidity content. A statistical analysis gives an excellent correlation coefficient of $\mathrm{R}=0.994$. The measurement value at $100 \%$ humidity content was obtained via a measurement of isotonic $\mathrm{NaCl}$ solution $(0.9 \%$ salinity). As can be seen in Fig. 5 , its permittivity also fits to the linear regression. It is important to note, that the presence of electrolytes in water causes a decrement of the relative permittivity [7]. Since the serum in the brain has a very similar concentration of electrolytes to the isotonic $\mathrm{NaCl}$ solution [8], this measurement verifies our findings. In the next step, a nondestructive method for measuring the complex permittivity is explored, to avoid the necessity of tissue-sampling.

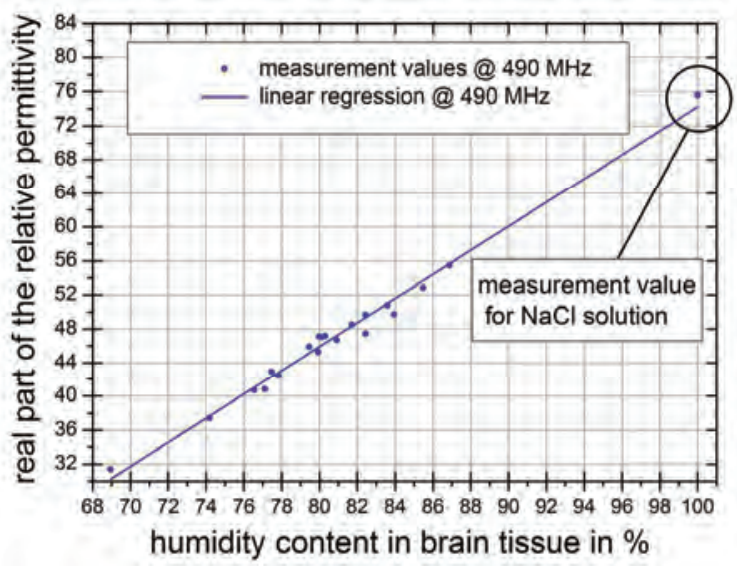

Fig. 5: Linear regression between the real part of the permittivity, measured with our system, and the humidity content, measured with the thermo gravimetric reference system, in the brain tissue at $490 \mathrm{MHz}$

\section{Open-ended coaxial line}

Fig. 6 depicts a schematic of an open-ended coaxial measurement. The specimen terminates the open-ended coaxial line, consisting of an inner and outer conductor and a flange. From an electromagnetic simulation with CST MICROWAVE STUDIO ${ }^{\circledR} 2013$ it can be seen, that the interaction of a TEM-wave with the specimen causes an electromagnetic field in the specimen. The penetration depth of this field is just a few millimeters, so that no special requirements exist for the measurement setup, especially for the material of the preparation table.

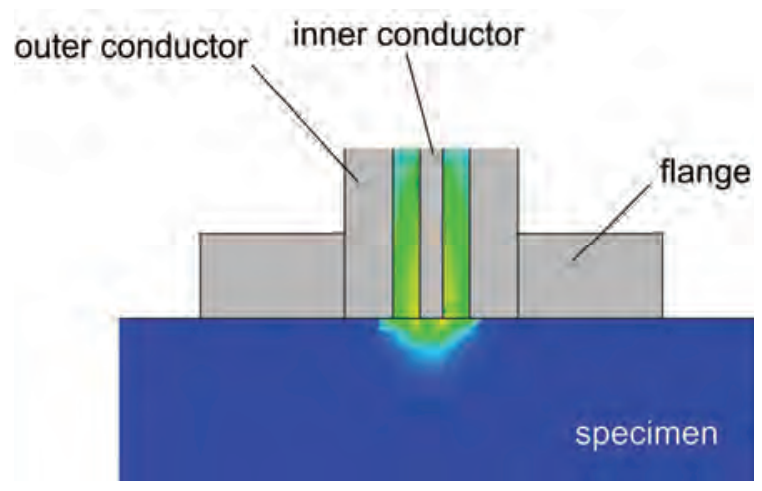

Fig. 6: Schematic of an open-ended coaxial line with simulated field in the specimen.

The field distribution can now be used to calculate the dielectric constant of the specimen Therefore the specimen is modeled as an equivalent circuit, terminating the coaxial line. In [9], it is shown that for low frequencies the admittance $y$ represents a dielectric, terminating an open-ended coaxial line. It can be written as

$$
y=\varepsilon_{r}+\xi \varepsilon_{r}^{2},
$$


where $\xi$ is an unknown constant, depending on the frequency and the dimensions of the aperture. It can be easily found through simple calibration measurements of the reflection coefficients $\Gamma$ of a short circuit and three specimens with known permittivity. The admittance $y$ of a specimen with unknown permittivity can be calculated from a measurement of the corresponding reflection coefficient $\Gamma$ as described in [9]. With $\xi$ and $y$ the unknown permittivity can be calculated using (6).

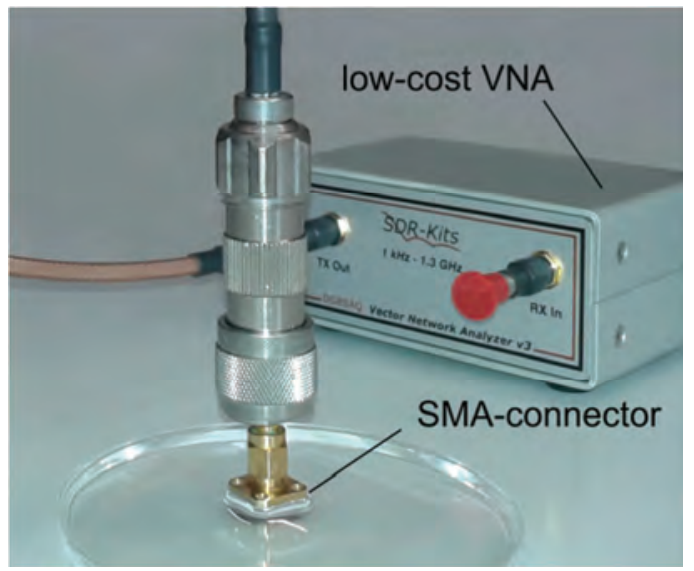

Fig. 7: Measuring setup with VNA (VNWA3, SDRKits) and open-ended coaxial probe

\section{Measurement setup with open-ended coaxial probe}

Fig. 7 depicts the experimental setup for measuring the calibration liquids. The setup consists of the low-cost vector-network analyzer (VNA) VNWA3 (SDR-Kits) already used for the coaxial measurement and the open-ended probe. For first experiments, the probe is just a standard SMA-connector with a mounting flange, which was slightly modified by flattening the inner conductor at the open end, see Fig. 8.

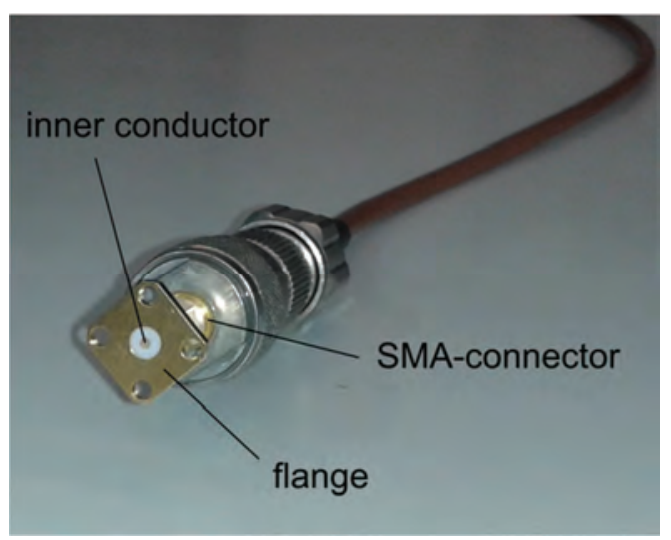

Fig. 8: Modified SMA-connector with mounting flange used as an open-ended coaxial probe

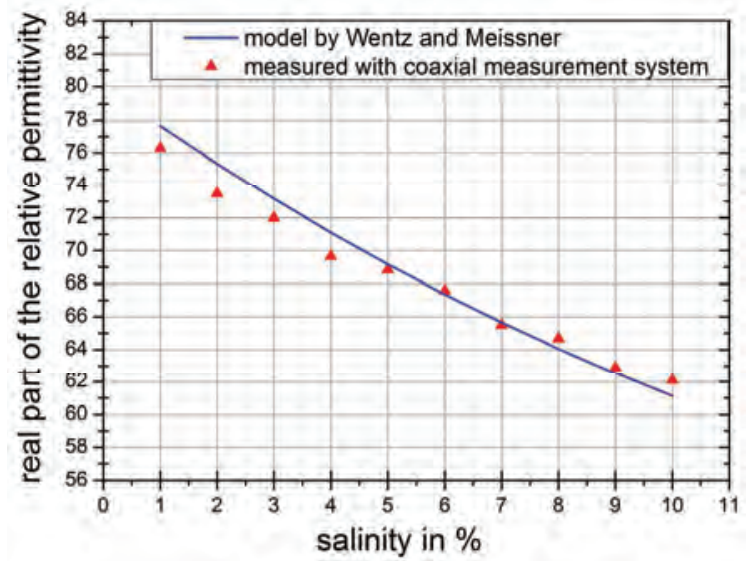

Fig. 9: Coaxial measurement of the permittivity of saline solution at $500 \mathrm{MHz}$ compared with a model by Wentz and Meissner

To verify the results from the open-ended coaxial measurements, we performed measurements with the previous described coaxial measuring chamber on saline solutions with salinity from $1 \%$ to $10 \%$ at a frequency of $500 \mathrm{MHz}$. The results are depicted in Fig. 9. A very good accordance between the measurement values and the salinity model for the dielectric constant proposed by Wentz and Meissner in [10] can be seen.

As described in [11], the permittivity of the calibration materials should resemble the specimens' permittivity. To calibrate the openended coaxial probe, we used the following dielectrics: Distilled water with a literature value for the complex permittivity at $500 \mathrm{MHz}$ of $\varepsilon_{r}=80.13-2.3 j$ [10], saline solution with a salinity of $4 \%$ and a value for the complex permittivity at $500 \mathrm{MHz}$ of $\varepsilon_{r}=71.102-196.08 j$ according to the model by Wentz and Meissner, as well as an open $\left(\varepsilon_{r}=1\right)$ and a short circuit. Fig. 10 shows the results from the measurement of saline solution with the open-ended coaxial probe, compared to measurement results with the coaxial measurement system and the salinity model of Wentz and Meissner. As can be seen, the measured values fit well to the theoretical values. Thus, it can be concluded, that it is possible to determine the permittivity of brain tissue very exactly with this method, if materials in the permittivity range from 30 to 60 are found for an appropriate calibration process. 


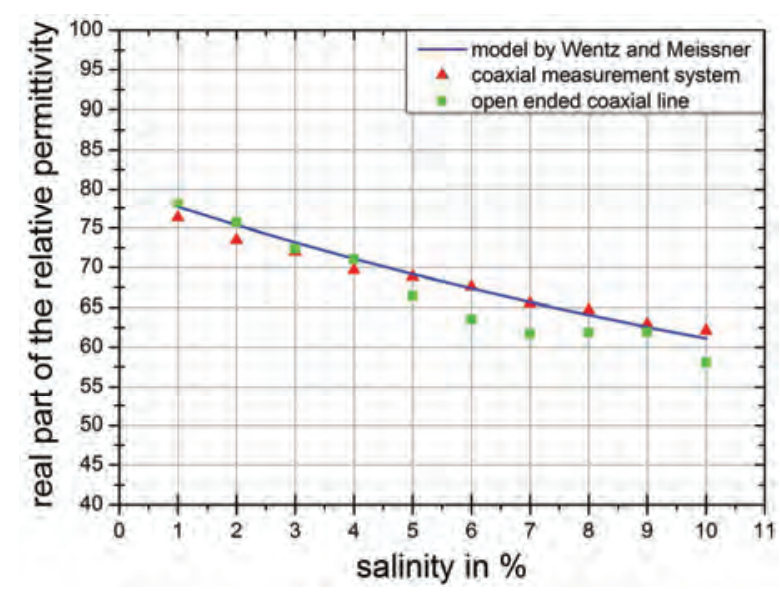

Fig. 10: Open-ended coaxial measurement of the permittivity of saline solution at $500 \mathrm{MHz}$ with revised calibration.

\section{Conclusion}

In this paper we present the results from permittivity measurements in human brain tissue with a coaxial measurement system, which results in a linear regression with an excellent regression coefficient between the real part of the permittivity and the humidity content in the brain tissue. The humidity content was measured with a commercially available thermo gravimetric reference system. The coaxial measurement system was also verified with measurements on saline solutions, where the findings matched well with the theoretical results of a permittivity model. Subsequent, a nondestructive open-ended coaxial method was tested for determining the humidity content in brain tissue. Therefore, the open-ended coaxial measurement system was calibrated with appropriate materials and tested with saline solutions. From this measurements it can be concluded, that it is possible to measure the relative permittivity and thus the humidity content of human brain tissue with the openended coaxial probe with high accuracy.

\section{References}

[1] Bogomolov DV, Dolzhanskiĭ OV, Kalashnikov DP, Luzin AV (2006), "Quantitative assessment of brain hydration in determination of death speed and type of tanatogenesis in forensic medical practice", Sud Med Ekspert. 49(1):14-6

[2] Collins Michael J., "Analytical method for determining volatiles, solids and solvent extractables", US4753889 A, 1988

[3] Kraszewski, A., "Microwave Aquametry," Microwave Conference, 1980. 10th European, vol., no., pp.48,58, 1980

[4] Nicolson, A.M.; Ross, G. F., "Measurement of the Intrinsic Properties of Materials by Time-Domain Techniques," Instrumentation and Measurement, IEEE Transactions on , vol.19, no.4, pp.377,382, 1970

[5] Baker-Jarvis, J.; Vanzura, E.J.; Kissick, W.A., "Improved technique for determining complex permittivity with the transmission/reflection method," Microwave Theory and Techniques, IEEE Transactions on , vol.38, no.8, pp.1096,1103, 1990

[6] Reinecke, Hagemeier, Schulte, Klintschar, Zimmermann, "Quantification of Edema in Human Brain Tissue by Determination of Electromagnetic Parameters", Sensors, 2013 IEEE (accepted for publication)

[7] Kraszewski A., "Microwave AquametryElectromagnetic Wave Interaction with WaterContaining Materials", New York: IEEE Press, 1996

[8] Schmidt R. F., Thews G., "Human Physiology", Springer, 1989

[9] Meissner, T.; Wentz, F.J., "The complex dielectric constant of pure and sea water from microwave satellite observations," Geoscience and Remote Sensing, IEEE Transactions on , vol.42, no.9, pp.1836,1849, 2004

[10] Staebell, K.F.; Misra, Devendra, "An experimental technique for in vivo permittivity measurement of materials at microwave frequencies," Microwave Theory and Techniques, IEEE Transactions on , vol.38, no.3, pp.337,339,1990

[11] Grady, M.; Wentworth, S.; Weller, T., "Improvements in cross ratio invariance techniques for coaxial probe dielectric measurements," Microwave Measurement Conference (ARFTG), 2012 79th ARFTG , vol., no., pp. 1,7, 2012 